\title{
Infrared Achromatic Phase Shifters using Modulated Total Internal Reflection
}

\author{
Dimitri Mawet ${ }^{a}$, Cédric Lenaerts ${ }^{b}$, Pierre Riaud $^{a}$, Denis Vandormael $^{c}$, Jérome Loicq $^{c}$, \\ David Verstraeten $^{c}$, Karl Fleury ${ }^{c}$, Serge Habraken ${ }^{b, c}$ and Jean Surdej ${ }^{a}$ \\ ${ }^{a}$ Institut d'Astrophysique et de Géophysique de Liège (IAGL), University of Liège, \\ 17 Allée du 6 Août, 4000 Sart Tilman, Belgium; \\ ${ }^{b}$ HOLOLAB, Department of physics, University of Liège, \\ 17 Allée du 6 Août, 4000 Sart Tilman, Belgium; \\ ${ }^{c}$ Centre Spatial de Liège (CSL), Av. du Pré-Aily, 4031 Angleur, Belgium.
}

\begin{abstract}
We propose a new family of achromatic phase shifters that uses the modulated total internal reflection (TIR) phenomenon. These components can be seen as enhanced Fresnel rhombs for infrared applications like nulling interferometry and polarimetry. The TIR phenomenon comes with a differential phase shift between the polarization components of the incident light. Modulating the index transition at the TIR interface allows compensating for the intrinsic material dispersion in order to make the subsequent phase shift achromatic over broad bands. The modulation can be induced by a thin film of a well-chosen medium or a subwavelength grating whose parameters are specially optimized. We present results from theoretical simulations together with preliminary fabrication outcomes.
\end{abstract}

Keywords: Nulling interferometry, Achromatic Phase Shifters, total internal reflection, thin films, subwavelength gratings

\section{INTRODUCTION}

Direct detection and characterization of faint sources around bright astrophysical objects like stars or Active Galactic Nuclei is very difficult due to the large flux ratio between them. For example, an Earth-like exoplanet is typically $\approx 10^{9}$ times fainter than its host star in the visible spectrum, $\approx 10^{6}$ in the thermal infrared. Infrared nulling interferometry proposed by R. Bracewell in $1978^{1}$ appears to be the most promising technique to achieve the high angular resolution and high dynamic range required to allow the ambitious detection of the first exobiological tracers. The nulling interferometry technique consists in adjusting the phases of the beams coming from various telescopes (two in the most simple configuration) to produce a fully destructive interference on the optical axis. The quality of the destructive interference, or the so-called null depth (ND) relies on the optical components ability to induce a very precise phase shift (e.g. $\pi$ ) and a very low amplitude mismatch over the considered wavelength range.

Unfortunately, searching biomarkers in the exoplanet atmospheres requires spectroscopic characterization over large spectral bands. For example, the DARWIN Infrared Space Interferometer ${ }^{2}$ considered by the European Space Agency (ESA) will operate in a wavelength band between 6 and 18 microns. The huge flux ratio between the parent star and the planet therefore requires unprecedented high performance broadband phase shifters. For that, we propose to modulate the total internal reflection (TIR) interface of rhombohedra by means of subwavelength diffractive optical elements or thin films. These original components fit in the technological evolution of the well-known Fresnel rhomb technology. ${ }^{3}$ Our theoretical calculations using the Rigorous Coupled Wave Analysis point to promising results.

Further author information: (Send correspondence to D. Mawet)

D. Mawet: E-mail: mawet@astro.ulg.ac.be, Telephone: +32 (0)4 3669757

C. Lenaerts: E-mail: Cedric.Lenaerts@ulg.ac.be, Telephone: +32 (0)4 3663773

Advances in Stellar Interferometry, edited by John D. Monnier, Markus Schöller, William C. Danchi, Proc. of SPIE Vol. 6268,

62682J, (2006) -0277-786X/06/\$15 - doi: 10.1117/12.671572 


\section{PRINCIPLE}

The TIR phenomenon comes with a differential phase shift between the vectorial TE and TM polarization components. This vectorial phase shift takes the following form ${ }^{4}$

$$
\Delta \phi_{T E-T M}=2 \arctan \left[\frac{\sqrt{\sin ^{2} \theta-n_{t i}^{2}}}{n_{t i}^{2} \cos \theta}\right]-2 \arctan \left[\frac{\sqrt{\sin ^{2} \theta-n_{t i}^{2}}}{\cos \theta}\right]
$$

where $\theta$ is the angle of incidence, greater or equal to $\theta_{c}$, the critical angle defined as $\sin \theta_{c}=n_{t i}=n_{t} / n_{i}$ and where $n_{i}$ and $n_{t}$ are the refractive indices of the incident and emergent media, respectively. This property is exploited in the well-known Fresnel rhombs. We demonstrate that engraving a subwavelength grating on the TIR interface or simply depositing a single thin layer of a well chosen material leads to a significant improvement over the Fresnel rhomb technology which is limited by the intrinsic index dispersion of the bulk material used.

\subsection{Total internal reflection grating}

As already mentioned, the principle of the total internal reflection grating achromatic phase shifter (TIRG APS) is to use a subwavelength grating in the TIR incidence condition (see Fig. 1).

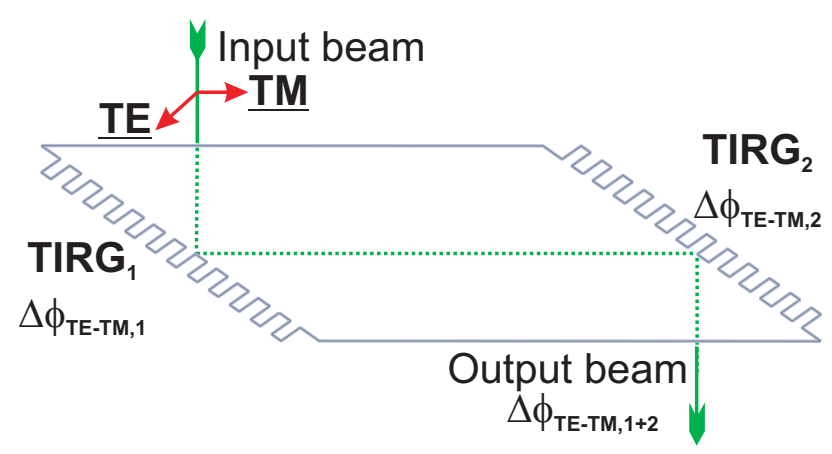

Figure 1. Schematic of the TIRG APS component. The TIRG APS is analog to a Fresnel rhomb which TIR interfaces are engraved with an optimized subwavelength grating. A TIRG APS component calculated for a $\pi$ phase shift can possess for example two TIR interfaces, each providing a $\pi / 2$ phase shift such that the resultant is $\Delta \Phi_{T E-T M, 1+2}=$ $\Delta \Phi_{T E-T M, 1}+\Delta \Phi_{T E-T M, 2}=\pi / 2+\pi / 2=\pi$. Such a component is to be inserted in each interferometer arm and orthogonally from one another.

Indeed, when the period of a grating becomes smaller than the wavelength of the incident light, it does not diffract light as usual in the sense that only the zeroth transmitted and reflected orders are allowed to propagate outside the modulated regions, leaving wavefronts free from any further aberrations*. Of course, in the TIR configuration case, the only order allowed to propagate is the specularly reflected one.

Interaction between the grating and the vectorial electromagnetic field leads to interesting effects on the phase and amplitude of the external propagating fields. Indeed, one dimensional subwavelength gratings, i.e. gratings only modulated along one dimension, turn out to be artificially birefringent. It means that the structure can be associated with two so-called effective indices, one for each polarization component TE and TM. These effective indices, $n_{T E}$ and $n_{T M}$ are totally dependent on the grating and incidence geometries (see Fig. 2). As the geometry can be controlled, one can really speak of refractive index engineering. Subwavelength gratings have recently been proposed in their transmission configuration for implementation into phase mask coronagraphs: ${ }^{5,6}$ the so-called dispersion of form birefringence is used as a tool to device integrated masks with high performances (achromaticity).

Phenomenologically, the use of subwavelength gratings in TIR incidence implies that the field interacts with the structure only by means of its evanescent waves. The phase shift between the orthogonal polarization

\footnotetext{
${ }^{*}$ For this reason, such gratings are often known as zero-order gratings (ZOG).
} 


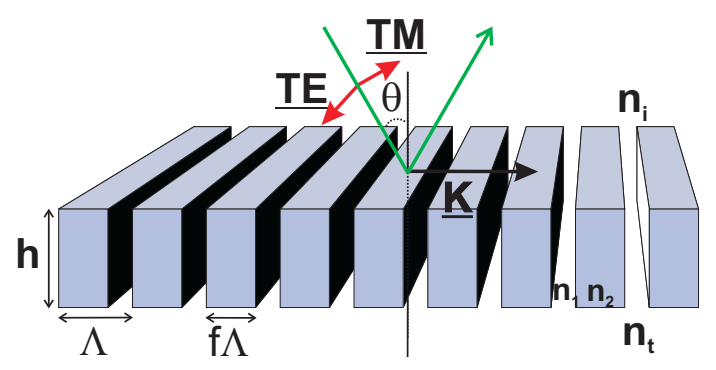

Figure 2. Schematic representation of a subwavelength grating. The main parameters of the structure are: the grating vector $|\underline{K}|=2 \pi / \Lambda$, perpendicular to the grating lines, with $\Lambda$ being the spatial period, the grating depth $h$ and the filling factor $f$, such that $f \Lambda$ is the width of the grating ridges. $\underline{T E}$ and $\underline{T M}$ are the vectorial orthogonal polarization components of the $\theta$-incident light. $n_{i}$ and $n_{t}$ are the refractive indices of the incident and emergent (transmitting) media, respectively. $n_{1}$ and $n_{2}$ are the refractive indices of the grating itself (in our case, $n_{2}=n_{i}$ and $n_{1}=n_{t}$ ).

components arises due to the fact that their associated vanishing fields penetrate more or less deeply into the modulated region, inducing a pseudo-optical path difference responsible for the phase shift. This differential skin effect can be explained by the anisotropy induced by the TE-TM zeroth order effective indices. The achromaticity of the subsequent retardation can be understood from the particular grating-induced artificial dispersion of the form birefringence but, most of all in the reflection mounting case, by the complex interaction between the higher order vanishing modes. ${ }^{7}$

With a precise control the grating parameters, the induced vectorial phase shift can be made as nearly achromatic as possible. Achromatic means in this case that the phase shift value remains constant over the considered wavelength range. As the leading application of the TIRG APS component concerns nulling interferometry (the TIRG APS high performances could as well be used in other applications like polarimetry), we chose to optimize the grating design with the null depth as the figure of merit. The null depth somehow is the quantitative measure of the darkness of the destructive interference. The TIR configuration ensures a $100 \%$ efficiency for the back reflected light whatever the polarization, therefore no amplitude mismatches. The figure of merit to be minimized consequently resumes to $\operatorname{ND}(\lambda)=\sigma^{2} / 4$ where $\sigma(\lambda)$ is the phase shift error standard deviation with respect to the nominal value of $\pi$ (any other value is possible). This means that the null depth is directly proportional to the variance of the phase shift, i.e. the achromaticity. The DARWIN specification allows a maximum null depth of $10^{-6}$ over the 6-18 $\mu \mathrm{m}$ wavelength range, corresponding roughly to the contrast ratio between the star and the earth-like planet in the thermal infrared. However, in pratice, we will always require better performances for the APS, i.e. a few $10^{-7}$ to account for other systematic errors in the interferometer.

\subsection{Total internal reflection thin film}

Instead of engraving a subwavelength grating onto the TIR interface, one can deposit a layer of a well-chosen foreign material. The principle of the so-called total internal reflection thin film achromatic phase shifter (TIRTF APS) is to make use of such a thin film coated with an appropriate thickness on the TIR interface. This principle has been known for quite a long time in the visible where $M g F_{2}$ thin films are commonly deposited on $B K 7$ or Silica rhombs to improve the angular and chromatic behavior of commercial Fresnel rhombs. ${ }^{8}$ The physical explanation of this is the same as for subwavelength gratings: the phase difference between the polarization components is influenced, or in other words, modulated by the differential skin effect engendered by the presence of the foreign optimized layer.

\subsection{Double rhomb configuration}

Single Fresnel rhombs such as illustrated in Fig. 1 are known to be very sensitive to incidence varations (at the arcmin level). This drawback would be penalizing in our application since the thermal infrared interferometric beam is likely to slightly diverge because of diffraction while somewhat wandering around because of vibrations. For this reason, we chose the double rhomb configuration for its known insensitivity to incidence variations. ${ }^{3}$ Indeed, the pairs of reflections in the two rhombs are complementary (see Fig. 3); an increase in the first two 
TIR angles due to departure from nominal incidence leads to a decrease in the last two angles. Thus if the phase shift varies linearly with the TIR angle, the retardance changes are cancelled. Therefore a wider range of incidence angle variations can be tolerated: upto several degrees.

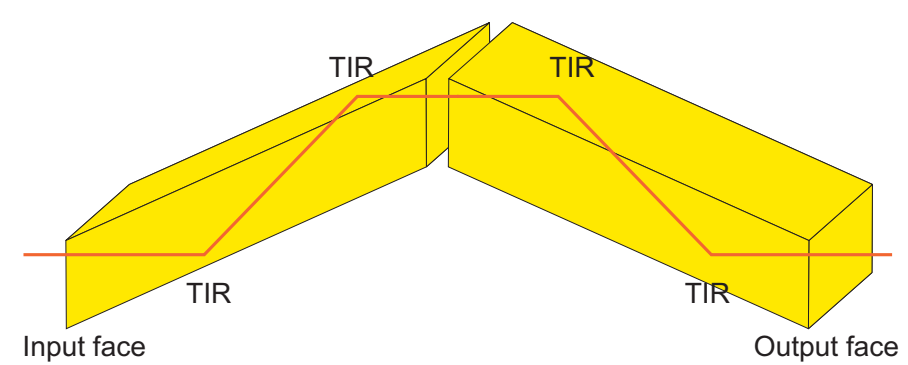

Figure 3. Here is shown the double rhomb configuration. This geometry allows incidence angle variations to be compensated by the angle complementarity between the two rhombs.

It is to be noted that in the double rhomb configuration, the number of subwavelength gratings to be imprinted (resp. thin film to be deposited) on the TIR interface may be limited to only two out of four. Indeed, the dispersion compensation artificially introduced by the modulated interface is sufficient to reach the specifications.

\section{THEORETICAL RESULTS}

We have performed "simplex" optimization of the component parameters for the DARWIN wavelength bands: 6 to 11 microns, 11 to 18 microns, possibly 6 to 18 microns or 6 to "above 11 microns", i.e. as far as the bulk material intrinsic transparency permits it. We had then to choose among a restricted list of infrared materials: we will present simulation results using Diamond (CLD, Carbon like Diamond), Zinc Selenide (ZnSe), Zinc Sulfide $(Z n S)$, Cadmium Telluride $(C d T e)$ and Germanium $(G e)$. We had to discard more common materials like Silicon $(S i)$ and Gallium Arsenide $(G a A s)$ for their strong multi-phonon absorption features beginning between 8 and 12 microns. Moreover, the choice for the bulk material of the rhomb is severely limited since it has to be perfectly transparent up to at least 11 microns, preferably 18 microns and of course available in large ingots of very good optical quality (low residual stress birefringence, low impurity, ...). For this reason, we have concentrate on $Z n S e, C d T e$ and $G e$ for the bulk material. It is to be emphasized that $C d T e$ is the only one perfectly clear up to 18 microns $^{\dagger}$.

Table 1. Temperature-dependant coefficients for material index representations. CLD stands for Carbon like Diamond.

\begin{tabular}{cccccc}
\hline Coeff. & CLD & ZnSe & ZnS & CdTe \\
\hline A & 1 & 1 & $5.608 \times 10^{-5} T+2.282$ & $-2.973 \times 10^{-4} T+3.8466$ & $-6.040 \times 10^{-3} T+11.05128$ \\
B & 0.3306 & 4.46395 & $-8.671 \times 10^{-6} T-1.563 \times 10^{-2}$ & $8.057 \times 10^{-4} T+3.2215$ & $9.295 \times 10^{-3} T+4.00536$ \\
C & 0.030625 & 0.0404 & $5.549 \times 10^{-7} T+2.067 \times 10^{-3}$ & $-1.10 \times 10^{-4} T+0.1866$ & $-5.392 \times 10^{-4} T+0.599034$ \\
D & 4.3356 & 0.46132 & $2.597 \times 10^{-8} T-1.714 \times 10^{-4}$ & $-2.160 \times 10^{-2} T+12.718$ & $4.151 \times 10^{-4} T+0.09145$ \\
E & 0.011236 & 0.1538 & $-9.798 \times 10^{-10} T+2.884 \times 10^{-6}$ & $-3.160 \times 10^{1} T+18753$ & 0 \\
F & 0 & 2.88289 & 0 & 0 & 0 \\
G & 0 & 2213.5 & 0 & 0 & 0 \\
\hline
\end{tabular}

These selected materials are common in infrared applications, they cover a large refractive index spectrum $(n=2-4)$ and their etching processes are well known for most of them. The indices and spectral dispersions

\footnotetext{
${ }^{\dagger}$ Note that $Z n S e$ and $Z n S$ can be used as the layer materials in their phonon absorption ranges since their working thicknesses are very thin and the subsequent absorptions therefore negligible.
} 
for Diamond, ${ }^{9} \mathrm{ZnSe},{ }^{10} \mathrm{ZnS},{ }^{11} \mathrm{CdT} \mathrm{e}^{11}$ and $G e^{11}$ will be taken from the following relations

$$
\begin{gathered}
n_{\text {Diamond }, Z n S e, C d T e, G e}(\lambda, T)=\left(A+\frac{B \lambda^{2}}{\lambda^{2}-C}+\frac{D \lambda^{2}}{\lambda^{2}-E}+\frac{F \lambda^{2}}{\lambda^{2}-G}\right)^{1 / 2} \\
n_{Z n S}(\lambda, T)=\left(A+B \lambda+C \lambda^{2}+D \lambda^{3}+E \lambda^{4}\right)^{1 / 2}
\end{gathered}
$$

The corresponding coefficients are given in Table 1 . We will assume in the following a working temperature $T=100 \mathrm{~K}$, keeping in mind that corrections are needed if $T$ is different. Note that $\lambda$ is expressed in microns in all representations. To simulate grating responses in the subwavelength domain, scalar theories of diffraction dramatically fail. The vectorial nature of light must be taken into account implying a resolution of the Maxwell equations. For that, we used an algorithm based on the Rigorous Coupled Wave Analysis ${ }^{12}$ (RCWA) for the simulations of the detailed grating behavior. RCWA is also called the Fourier modal method because it is based on Fourier decompositions of the physical characteristics of the grating and fields so that the resolution takes place in the frequency space.

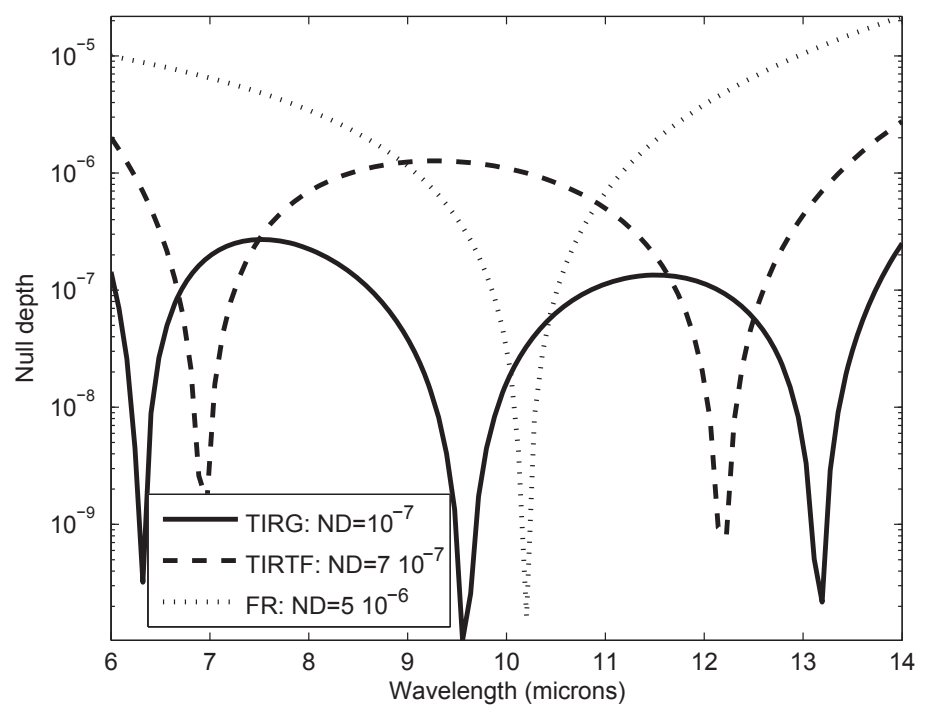

Figure 4. ZnSe double rhomb APS: comparison between Fresnel Rhomb (FR) with non-treated TIR interfaces, TIR thin film (TIRTF) and TIR grating (TIRG). More than substantially lowering the global (mean) null depth over the considered wavelength range, the TIRTF and the TIRG solutions significantly decrease the strong leakage at its edges, inevitable with the FR solution.

\subsection{ZnSe Rhomb}

This solution is the most practical one since $Z n S e$ is available in large quantities/volumes at a relatively low cost. Some comments about the results displayed in Table 2: first of all, it is to be noted that the $Z n S e$ bare Fresnel rhomb (without any ZOG or coating on the TIR interfaces) is not performing well enough for the DARWIN specification (see Fig. 4, dotted line). However, depositing a foreign material at the TIR interface or imprinting a subwavelength grating onto it allows overcoming this limitation at least in the limited $6-11 \mu \mathrm{m}$ wavelength range. The performance is somewhat degradated in the larger $6-14 \mu \mathrm{m}$ range, but still comfortably in the specifications for the subwavelength grating solution (Fig. 4, continuous line) while somewhat at the limit for the thin film one (Fig. 4, dashed line). Note that in this latter case, Carbon like Diamond would be particularly appropriate as the layer material. 
Unfortunately, the double rhomb configuration lengthens the optical path in the material. For instance, in the case of the ZnSe TIRG APS designed for operation between 6 and 14 microns, the working angle is 1.137 radians leading to a physical path of $17.27 \mathrm{~cm}$ inside the material. In the second DARWIN band, such a long path is penalizing since $Z n S e$ begins its phonon absorption around 14 microns: the absorption coefficient ${ }^{11} k$ is equal to $4.2410^{-6}$ at $300 \mathrm{~K}$, leading to an absorption of about $50 \%$, reducing to $2.5210^{-6}$ at $100 \mathrm{~K}$, giving an aborption of $30 \%$, which remains acceptable.

\subsection{CdTe Rhomb}

Availability of $C d T e$ ingots is more limited than $Z n S e$ ones. Polishing of $C d T e$ is also quite delicate but currently under evaluation for improvement by several manufacturers. Moreover, the price is higher but $C d T e$ nevertheless appears to be more than a viable solution ${ }^{\ddagger}$. Indeed, theoretical results of the expected final component performance are very good, better than with $Z n S e$. First of all, the $C d T e$ bare Fresnel rhomb solution is worth considering: theoretical results are within the DARWIN specifications for the first band but unfortunately not for the second one. However, depositing a thin film of a foreign material ( $Z n S e$ or Diamond) overcomes this limitation. As far as the subwavelength grating solution is concerned, results are comfortably in the specifications for both bands.

Let us emphasize that there exists a most interesting solution achromatizing the whole DARWIN wavelength range from 6 to 18 microns with only one component: an optimized double rhomb in $C d T e$ modulated with a subwavelength grating or even with a thin $Z n S e$ or Diamond film on at least two of the four TIR interfaces (see Table 2).

\subsection{Ge Rhomb}

Germanium is a very well suitable material for classical Fresnel rhombs since its dispersion is extremely low. ${ }^{11}$ Theoretical results for the bare Fresnel rhomb are excellent: $\mathrm{ND}=410^{-9}$ over the $6-11 \mu \mathrm{m}$ wavelength range. Adding a ZOG at the TIR interface further improves this already excellent behaviour. However, Ge is unfortunately not transparent in the second DARWIN band, making it a partial solution only.

Table 2. Null depths for the optimal Fresnel rhomb, TIRG APS, TIRTF APS configurations for the selected materials. CLD stands for Carbon like Diamond.

\begin{tabular}{cccc}
\hline Material/band & Fresnel rhomb & TIRG $\boldsymbol{A P S}$ & TIRTF APS \\
\hline ZnSe $/ 6-11 \mu \mathrm{m}$ & $1.6 \times 10^{-6}$ & $1 \times 10^{-8}$ & CLD $/$ ZnS layer: $1 \times 10^{-7}$ \\
ZnSe $6-14 \mu \mathrm{m}$ & $6 \times 10^{-6}$ & $1 \times 10^{-7}$ & CLD layer: $7 \times 10^{-7}$ \\
$\mathrm{CdTe} / 6-11 \mu \mathrm{m}$ & $2 \times 10^{-7}$ & $2 \times 10^{-9}$ & CLD $/ Z n S e / Z n S$ layer: $1 \times 10^{-8}$ \\
$\mathrm{CdTe} / 11-18 \mu \mathrm{m}$ & $8 \times 10^{-7}$ & $1 \times 10^{-8}$ & CLD $/ Z n S e / Z n S$ layer: $4 \times 10^{-8}$ \\
$\mathrm{CdTe} / 6-18 \mu \mathrm{m}$ & $1.6 \times 10^{-6}$ & $1 \times 10^{-7}$ & CLD $/ Z n S e / Z n S$ layer: $4 \times 10^{-7}$ \\
\hline
\end{tabular}

\section{IMPLEMENTATION}

Implementation of the vectorial phase shift in a nulling interferometer is straightforward. Considering two strictly identical components belonging to the two distinct interferometer arms, rotated by ninety degrees around the optical axis and from one another, then the potentially-interfering parallel polarization states are two by two in phase opposition. It must be noted that there is a strong constraint on the alignment of the components around the optical axis. Let $\Delta \chi$ be the misalignement angle. $\Delta \chi$ is directly related to the null depth $N D$ : $N D=$ $(1+\sin \Delta \chi)(1-\cos \Delta \chi) / 2$. Thus, in order to fulfill the constraint $N D=10^{-6}$, we must reach $\Delta \chi \leq 6$ arcmin.

Departure from nominal zero optical path length between the two components belonging to the two interferometer arms due to manufacturing/surfacing errors and/or differential thermal dilatations can be compensated

\footnotetext{
${ }^{\ddagger}$ It is to be noted that CSL possesses a $C d T$ e growing facility. Samples for our application are being grown and will soon be qualified (dispersion and thermo-optic coefficients).
} 
either by slightly tilting the rhombs or making use of beamsplitters with adjustable thicknesses if in the same material. It is to be noted that differences in the geometry of the paired rhomb segments, length for example, are not expected to be an issue provided that they are cut and polished together.

\section{TOLERANCING}

The fabrication of the TIRG APS is based on micro-electronic technologies. The first step consists in imprinting a grating mask in a resin coated on the chosen substrate material. The precision of this step is critical because it defines once and for all the lateral dimensions of the grating, in other words, the so-called feature line, i.e. the period multiplied by the filling factor. This pattern is then uniformly transferred in the substrate by an appropriate reactive plasma beam etching down to the desired depth. The fabrication has to be interactive to properly compensate for process errors. In situ monitoring ${ }^{13}$ is a possible solution but not the only one as we will see.

Let us take an example: a $Z n S e$ TIRG APS designed for the 6-14 $\mu \mathrm{m}$ wavelength range and assuming a predefined period of $900 \mathrm{~nm}$. Best solutions are searched using the "simplex" optimization method coupled to the RCWA algorithm with the following free parameters: the filling factor, the grating thickness and the incidence angle. Results of this optimization are displayed in Fig. 5 top left, where the optimal null depth is plotted versus the actual feature line, i.e. the product of the varying filling factor and the fixed period of $900 \mathrm{~nm}$. The corresponding adjustment of the optimal thickness is shown in Fig. 5, top right. Let us now fix the feature line to its optimal value, i.e. $210 \mathrm{~nm}$ and vary the thickness artificially, optimizing for each value the corresponding incidence. Results of this operation are shown in Fig. 5 middle left and right. The conclusion of this tolerance analysis is that, provided that there are interactions between measurements and manufacturing at each key step, the tolerances on the paramater definitions are very confortable, up to several tens of nanometers for the feature line and hundreds of them for the thickness. A posteriori correction is reported on the incidence angle which is very convenient since the double rhomb geometry still ensures at the macroscopic level the insensitivity to the entrance beam incidence configuration (input angle and beam divergence, for instance).

As far as the TIRTF APS component is concerned, tolerancing can be envisaged in another way. Since the parameter space is limited to two variables, i.e. the thickness of the layer and the angle of incidence, the working points can be traced in two-dimensional maps. From Fig. 5, bottom, we can conclude that the tolerance on the thickness is of several percents $(\approx 10 \%)$. It is to be noted that coatings are routinely deposited with a precision of $\approx 1-2 \%$ on the thicknesses (see Section 6 ).

Some specific microscopic and macroscopic tolerancing issues will not be considered in this paper. They are the subject of current researches, let us just cite some of them. The microstructure patterns are assumed to be rectangular. However, departure from this situation with trapezoidal profiles, for example, is currently considered since it is likely to emerge from the manufacturing process. The macrocomponents have also to be optimized for stray light dumping. Broadband anti-reflective treatments will be necessary as well as appropriate wedge angles at the different interfaces.

\section{MANUFACTURING}

\subsection{TIR Grating APS manufacturing}

The most appropriate method to manufacture the micro pattern into the rhomb material is based on nano-imprint and dry etching processes. The first one is necessary for masking the parts of the substrates to be protected during the etching step. Direct laser writing or mask exposure techniques are not applicable onto the rhomb facets since those classical micro-lithography methods are designed for accommodating thin and flat substrates only, such as wafers for micro electronics. The nano-imprint alternative allows replicating onto a thick substrate a mask previously originated by conventional photolithography onto a thin substrate.

Fig. 6 illustrates the principle. A master is originated by illuminating (with a contrasted pattern) a photoresist layer coated on a thin substrate. A photoresist relief structure is then obtained after a chemical process, as shown in Fig. 7. A mould is generated from that photoresist master and it is used to emboss a polymer layer deposited onto the rhomb facet. After demoulding, residual polymer is removed by an oxygen plasma in order to clear the substrate where it is supposed to be etched. 

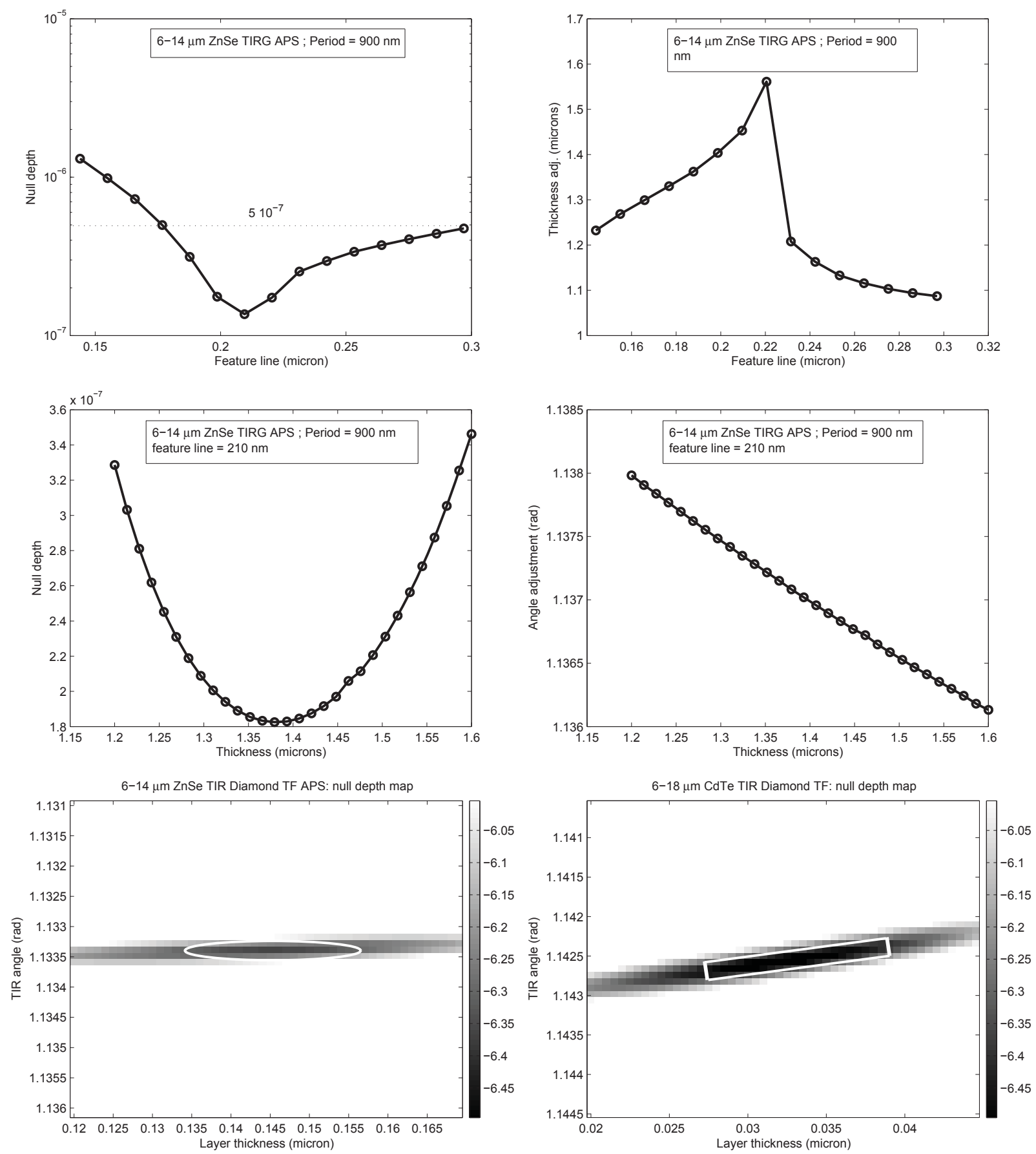

Figure 5. 6-14 $\mu \mathrm{m} Z n S e$ double rhomb APS with $900 \mathrm{~nm}$ period. Top left: optimized null depth vs feature line. Top right: thickness adjustment (optimized) vs feature line. Middle left: angle optimized null depth vs thickness for a fixed feature line of $210 \mathrm{~nm}$ (optimum). Middle right: corresponding angle adjustment (optimized) vs thickness. Bottom: twodimensional maps of the null depth ( $\log$ scale, $10^{-\alpha}$ ) according to the variables "thickness of the layer" and "incidence angle". Right: ZnSe TIRTF APS coated with Diamond for the 6 to 14 micron band. Left: CdTe TIRTF APS coated with Diamond for the 6 to 18 micron band. 
Light sensitive photoresist
Thin flat substrate

Light patterning of the photoresist layer

Chemical processing of the illuminated parts

\section{Microstructured mould fabrication}

Resulting microstructured mould
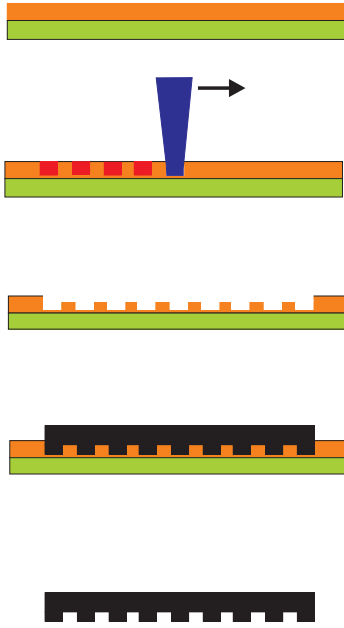

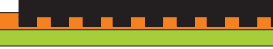
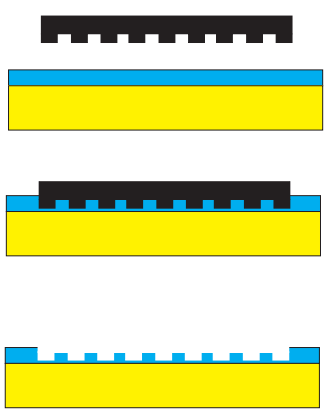

$+\mathrm{O}_{2}$ plasma

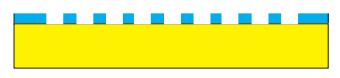

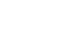

Figure 6. Nano-imprint lithography principle.

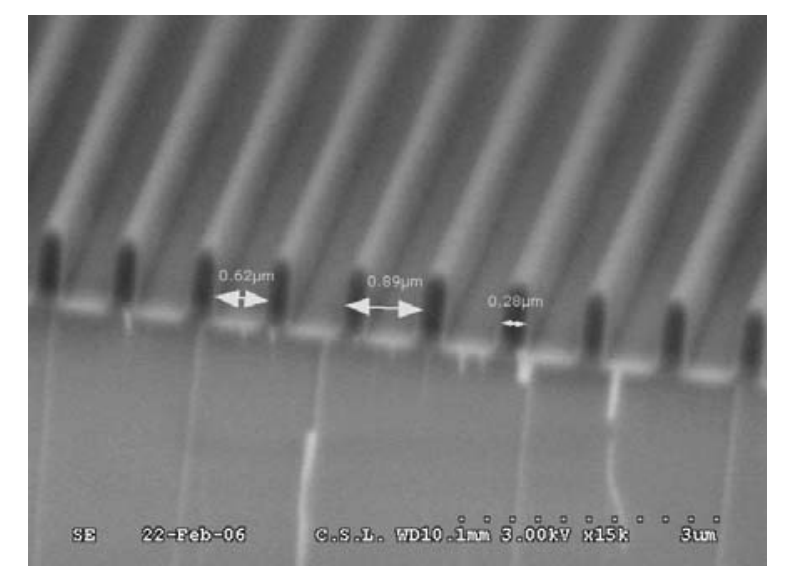

Figure 7. Master mould showing the micro pattern on photoresist to be transferred by nano imprint.

The remaining polymer pattern serves as a lithographic mask for the subsequent reactive plasma etching process. Reactive plasma beam etching (RPBE) makes use of both the ballistic effect and chemical reactivity of a beam of reactive ions to remove or create structures into a substrate. The various parameters (gas melanges, beam energy, beam incidence...) characterizing the etching process are optimized for the transfer into various materials. The interest of such a technique comes particularly from its high selectivity, the potential to efficiently etch one material and not another coexisting one. RPBE is widely used in the fabrication of micro-optical elements, micro-sensors and other micro-machines. As shown in Fig. 8, the infrared material $(Z n S e$ or $C d T e)$ is engraved using a selective chemistry which leaves intact the polymer mask but etches the infrared substrate. As already mentioned, the etched depth can be controlled with an interferometric in situ device.

This technique is still under development. The major difficulty is not the period definition $(\approx 1 \mu \mathrm{m})$ but the small feature size to be controlled simultaneously with the depth.

\subsection{TIR Thin Film APS manufacturing}

The thin film can be deposited with several coating technologies that have been developed and are currently in use in our labs. Classical evaporation is still used for simple coatings (like $Z n S e$ ), but advanced techniques allow preparing more compact layer coatings such as $Z n S$, for instance, which necessitates specific Ion Assisted 
Microstructure transfer
into the rhomb material by dry etching

Final structure after mask removal
+ reactive plasma
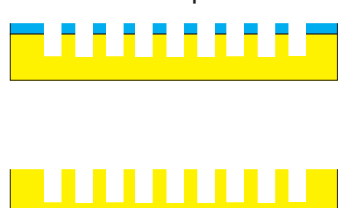

Figure 8. Pattern transfer from nano-imprinted mask into a substrate by dry-etching.

Deposition (IAD) techniques. ${ }^{14}$ It is to be noted that the well-known precision of the thickness control of these techniques can be an issue when one deals with very thin layers (typically less than $50 \mathrm{~nm}$ ). However, our tolerance analysis shows that it is still within reach.

As far as Diamond deposition is concerned, commercial treatments by chemical vapor deposition (CVD) are already available for $\mathrm{Ge}$ or $\mathrm{Si}$ substrates. To our knownledge, it has never been demonstrated on $Z n S e$ and $C d T e$ substrates. This may be due to the very high temperature needed for the Diamond growth, i.e. over 800 degrees Celsius. ${ }^{15}$ However, new promising approaches for Diamond deposition are currently studied to lower the growing temperature. Good results have been reported with temperature around 400 degrees Celsius. ${ }^{16}$ In the case of CVD Diamond, thickness control is ensured by the relatively slow growing rate, i.e. $\approx 200 \mathrm{~nm}$ per hour.

\section{CONCLUSION}

To summarize, we have presented a new concept of APS for thermal infrared radiations relying on the modulated TIR phenomenon. The modulation can be induced either by engraved subwavelength gratings or deposited thin films. Theoretical results show remarkable improvements over the classical Fresnel rhomb configuration, always limited by the intrinsic dispersion of the bulk material used. In the framework of nulling interferometry space missions like DARWIN and TPF-I, ${ }^{17}$ prototype manufacturing is being considered in our team in collaboration with the "Centre Spatial de Liège".

We would like to conclude on the following comment. Historically, one of the needs of microstructured components was coming from the space environment. Indeed, the components have to operate at cycling temperature when they are in orbit. From this point of view, the use of optical components with added coatings in the thermal infrared is sometimes questionable: the adhesion and the differential expansion can affect the performance by accelerated ageing. We showed that alternative solutions are emerging but at the same time involves a technologic break-through. None of the specific technique is unique but the combination or synergy makes it very complex. A long learning phase is also required. Compared to micro-electronics industry, the processes are somehow similar or derived. However, the materials are not the same. A process optimization taking into account the knowledge of physical and chemical properties is required. The University environment is helpful to develop those techniques, with industrial supports.

\section{ACKNOWLEDGMENTS}

The first author acknowledges the financial support of the Belgian "Fonds pour la formation à la Recherche dans l'Industrie et dans l'Agriculture". The authors also acknowledge the financial support of ESA.

\section{REFERENCES}

1. R. N. Bracewell, "Detecting nonsolar planets by spinning infrared interferometer," Nature 274, pp. 780-781, Aug. 1978.

2. A. Léger, J. Mariotti, B. Mennesson, M. Olliver, J. Puget, D. Rouan, and J. Schneider, "Could We Search for Primitive Life on Extrasolar Planets in the Near Future? - The DARWIN Project," Icarus 123, pp. 249-255, 1996. 
3. K. Rochford, A. Rose, P. Williams, C. Wang, I. Clarke, P. Hale, and G. Day, "Design and performance of a stable linear retarder," Appl. Opt. 36, pp. 6458-6465, 1997.

4. M. Born and E. Wolf, Principles of Optics, Cambridge University Press, 7 ed., 1999.

5. D. Mawet, P. Riaud, J. Surdej, and J. Baudrand, "Subwavelength surface-relief gratings for stellar coronagraphy," Appl. Opt. 44, pp. 7313-7321, 2005.

6. D. Mawet, P. Riaud, O. Absil, and J. Surdej, "Annular Groove Phase Mask Coronagraph," Astroph. J. 633, pp. 1191-1200, Nov. 2005.

7. D. Mawet, C. Lenaerts, P. Riaud, J. Surdej, S. Habraken, and D. Vandormael, "Use of subwavelength gratings in TIR incidence as achromatic phase shifters," œ 13, pp. 8686-8692, 2005.

8. P. Clapham, M. Downs, and R. King, "Some applications of thin films to polarization devices," Appl. Opt. 8, pp. 1965-1974, 1969.

9. F. Peter $Z$ phys. 15, pp. 358-368, 1923.

10. W. J. Tropf, "Temperature-dependent refractive index models for BaF2, CaF2, MgF2, SrF2, LiF, NaF, KCl, ZnS and ZnSe," Opt. Eng. 34, pp. 1369-1373, 1995.

11. G. Hawkins, PhD Thesis, Spectral Characterisation of Infrared Optical Materials and Filters, The University of Reading UK, 1998.

12. M. Moharam and T. Gaylord, "Diffraction analysis of dielectric surface-relief gratings," J. Opt. Soc. Am. 72, pp. 1385-1392, 1982.

13. P. Lalanne, P. Pichon, P. Chavel, E. Cambril, and H. Launois, "Interferometric Characterization of Subwavelength Lamellar Gratings," Appl. Opt. 38, pp. 4980-4984, 1999.

14. F. Lemarquis, G. Marchand, and C. Amra, "Design and Manufacture of Low-Absorption ZnS YF 3 Antireflection Coatings in the 3.5 16-micron Spectral Range," Appl. Opt. 37, pp. 4239-4244, 1998.

15. P. Dore, A. Nucara, D. Cannav, G. De Marzi, P. Calvani, A. Marcelli, R. S. Sussmann, A. J. Whitehead, C. N. Dodge, A. J. Krehan, and H. J. Peters, "Infrared Properties of Chemical-Vapor Deposition Polycrystalline Diamond Windows," Appl. Opt. 37, pp. 5731-5736, 1998.

16. X. Xiao, J. Birrell, J. E. Gerbi, O. Auciello, and J. A. Carlisle, "Low temperature growth of ultrananocrystalline diamond," Journal of Applied Physics 96(4), pp. 2232-2239, 2004.

17. C. A. Beichman, N. J. Woolf, and C. A. Lindensmith, eds., The Terrestrial Planet Finder (TPF): a NASA Origins Program to search for habitable planets, JPL Publication 99-3, Pasadena, 1999. 